\title{
Increased TLR/MyD88 signaling in patients with obesity: is there a link to COVID-19 disease severity?
}

\author{
Ada M. Cuevas ${ }^{1} \cdot$ Jeanne M. Clark ${ }^{2} \cdot$ James J. Potter ${ }^{2}$ \\ Received: 27 July 2020 / Revised: 4 December 2020 / Accepted: 20 January 2021 / Published online: 26 February 2021 \\ (c) The Author(s), under exclusive licence to Springer Nature Limited 2021
}

\begin{abstract}
COVID-19 is a pandemic disease caused by a coronavirus, designed as SARS CoV-2, whose clinical presentation is widely variable, with most patients having mild or no symptoms, but others developing a malign disease with multi-organ failure and even death. Accumulating data from different populations have shown that obesity is a risk factor for a severe evolution of the disease, however, the mechanisms that explain this association are not clearly understood. An ominous evolution of COVID-19 has been attributed to an exacerbated inflammatory response, designed as "cytokine storm" with augmented production of cytokines/chemokines through the activation of toll-like receptors (TLR) by pathogen-associated molecular patterns, that triggers an inflammatory downstream response, mediated in part by the adaptor molecule, myeloid differentiation factor 88 (MyD88). Previous studies have reported an increased expression of MyD88 and TLRs in people with obesity, mainly in those with metabolic complications. Therefore, we hypothesize, that an underlying increased Myd88/ TLR signaling may predispose to patients with obesity to develop an exaggerated and dangerous inflammatory reaction against SARS CoV-2 infection, explaining at least in part, the higher severity of COVID-19. In addition, MyD88/TLR signaling in people with obesity could have a role in the development of several chronic diseases.
\end{abstract}

Starting in early 2020, a devastating pandemic, caused by a coronavirus designated as SARS CoV-2, began to affect most of the countries in the world. The clinical manifestations of the COVID-19 disease are variable, with most of the affected people having mild or even no symptoms, but $\sim 10-15 \%$ of the patients developing the more severe disease with acute respiratory distress syndrome (ARDS), hypoxia, multi-organ failure, and even death $[1,2]$.

Accumulating data indicate obesity is a risk factor for poor prognosis with infection, along with older age, male sex, and presence of chronic diseases, hematological malignancies, or immunological disorders. Notably, results from different populations have confirmed a significant and independent association between obesity and severe COVID-19 disease. A large cohort study from primary care electronic health records in England (with a total population

Ada M. Cuevas

acuevas@cammyn.cl

1 Center for Advanced Metabolic Medicine (CAMMYN), Santiago, Chile

2 The Johns Hopkins University, Baltimore, MD, USA of $17,425,445$ people and 5683 deaths) linked to COVID19 , reported an increased risk of death which increased with BMI (HR 1.27 for BMI $30-34.9 \mathrm{~kg} / \mathrm{m}^{2}$, increasing to 2.27 for $\mathrm{BMI} \geq 40 \mathrm{~kg} / \mathrm{m}^{2}$ ) [3].

Other data show that an ominous evolution of the COVID19 disease is related to an excessive inflammatory response, considered as a "cytokine storm", characterized by elevated levels of different cytokines and chemokines including tumor necrosis factor- $\alpha$ (TNF), interleukin $1 \beta$ (IL $\beta)$, granulocytecolony stimulating factor, interferon $\gamma$-induced protein 10 , monocyte chemoattractant protein-1, macrophage inflammatory proteins $1-\alpha$, Interleukin-6 (IL-6), and the chemokines CXCL10 and CCL2 which are associated with pulmonary inflammation and are also increased in other viral infections [4]. This over-inflammatory response induced by the virus is not restricted to the lung and has been implicated in the multi-organ failure in patients with more severe disease. Thus, although the immune response is vital for the control and resolution of SARS CoV-2 and other infections, in some cases it could lead to a pathogenic condition characterized by an exacerbated inflammatory and altered immune reaction. Therefore, it is important to elucidate which host factors could induce an exaggerated and harmful response. Another article supports the concept that virus-independent 
immunopathology is a primary mechanism underlying fatal Covid-19 suggesting that targeting of the dysregulated immune response warrants further investigation [5].

In COVID-19 disease, SARS CoV-2 binds to the host cells by the angiotensin-converting enzyme 2 , fusing to the membrane and releasing viral RNA [6]. The immune system recognizes pathogen-associated molecular patterns of the virus via pattern recognition receptors including a group of transmembrane proteins, named TLR, located on the cell surface (TLR 1,2,4,5,6) or in specialized endosomal compartments (TLR 3, 7, 8, and 9) [7]. It has been hypothesized that SARS CoV-2 is recognized by TLRs which sense viral RNA in the endosomes, triggering an inflammatory downstream response, mediated in part by the adaptor molecule, MyD88. Activation of MyD88, would lead to the activation of nuclear transcription factors, such as nuclear factor-kB, cyclic AMP response element-binding protein, and activator protein-1 ensuring the production of pro-inflammatory cytokines, such as TNF and IL-1, chemokines, and other soluble mediators with subsequent inflammation and leukocyte accumulation [8]. A recent study suggested that a boosted pro-inflammatory response via TLR7/8 recognition could mediate the lung injury caused by the virus SARS CoV-2 [9]. Besides, recent studies have suggested that also TLR4 could be involved in the pathological inflammatory response induced by SARS CoV-2 $[10,11]$. Although SARS CoV-2 is unlikely to activate TLR4 directly, as TLR4 respond to bacteria, some damage-associated molecular pattern, such as oxidized phospholipids could be responsible for the activation of TLR4 by SARS CoV-2.

It is well known that people living with obesity, mainly with abdominal visceral fat accumulation, exhibit low-grade chronic systemic inflammation, which plays a role in the development of metabolic complications, including insulin resistance, dyslipidemia, reduced fatty acid oxidation, and oxidative stress. These are thought to be the common underlying pathophysiologic mechanisms for the development of diabetes, cardiovascular disease, some types of cancer, non-alcoholic steatohepatitis, neurological diseases, and other co-morbidities associated with obesity [12]. Moreover, researchers have elucidated an important role for immune cells in the physiological dysfunction associated with obesity. Metabolic disturbances lead to immune activation in tissues, such as adipose tissue, liver, pancreas, endothelial cells, and others and individuals often present with elevated plasma markers of chronic low-grade inflammation.

In a previous study of patients with obesity who underwent bariatric surgery, we detected significantly higher expression of MyD88 in the subcutaneous adipose tissue among those with at least three components of the metabolic syndrome (hypertension, high glucose, high triglycerides, or low HDL cholesterol plasma levels), in comparison with matched patients (by sex, age, and BMI) without metabolic complications [13].
Moreover, the expression of MyD88 in the adipose tissue was significantly correlated with the expression of TLR4, suggesting an activated TLR/MyD88 signaling in the group of patients exhibiting metabolic complications.

A previous study also found a significantly increased expression of TLRs and MyD88 in the mononuclear cells and adipose tissue of overweight and obese patients compared to lean controls. Moreover, the expression of IL-6 and TNF $\alpha$ was also significantly elevated in obese compared to controls and significantly associated with TLR expression [14].

We hypothesize that people with obesity, especially excess abdominal/visceral fat and associated metabolic complications, have overexpression of MyD88 in the adipose tissue and perhaps in other cells and tissues (as immune cells), that triggers an exaggerated inflammatory response of the immune system when activated by Sars CoV-2, leading to a more severe and sometimes lethal progression of COVID-19. Two possible pathogenic pathways could be proposed: (1) An increased SARS CoV-2 entry to the adipocytes, through the ACE-2 receptors, (that are also over-expressed in the adipose tissue of patients with obesity), activates TLRs, triggering the TLR/ MyD88 signaling inflammatory cascade and/or (2) the higher expression of MyD88 detected in abdominal fat of patients with obesity could also be enhanced in other tissues, including the lung and endothelial cells, mediating the respiratory, and vascular damage produced by SARS CoV-2.

A recent study in an animal model of ARDS detected that the inhibition of TLR4/MyD88 signaling pathway might result in decreased edema and inflammation in the lungs, by suppressing the inflammatory response, which appears to be regulated by macrophage activation [15]. A similar study demonstrated that the inhibition of the TLR4/ MyD88 signaling pathway reduced the expressions of proinflammatory cytokines IL- $1 \beta$ and TNF- $\alpha$, thereby ameliorating the pro-inflammatory phenotype [16].

Other authors have proposed that TLRs agonists, including Imiquimod an immune-stimulator of TLR7, could be an effective therapeutic approach during the early stages of COVID-19 disease [17]. However, we hypothesized that in patients with an advanced and severe presentation of the disease (including obese patients), the use of TLR antagonists would have a beneficial effect by attenuating the deleterious over-inflammatory response triggered by the SARS CoV-2 infection.

Otherwise, other research done in murine primary liver cells, evidenced that corticosteroids shift the TLR response pattern from a pro-inflammatory to an anti-inflammatory state in a cell type-specific fashion resulting in downregulation of TLRs expression, suppression of pro-inflammatory and upregulation of anti-inflammatory cytokines [18]. This line of thinking is supported by a study of patients with tuberculous meningitis, which revealed that dexamethasone treatment significantly reduces the TLR4 and MyD88 expression in 
monocytes [19] and it has been evidenced a beneficial effect of dexamethasone in patients with COVID-19 [20].

Similarly, statins in addition to their cholesterol-lowering effects, are also known to reduce pro-inflammatory markers, including TNF $\alpha$, IL-1, IL-6, CRP, and chemokines. Moreover, in a study using murine pro-B cells, it was shown that atorvastatin reduces pro-inflammatory markers through the inhibition of the TLR/MyD88/NFkB pathway in a MyD88 dependent manner [21]. Taken all together these studies indicate a key role of MyD88 in pro-inflammatory signaling and that reduced TLR/MyD88/NF signaling may provide the mechanism for the beneficial effects of steroids and statins in COVID-19.

In summary, people with obesity are at high risk of severe COVID-19 infection and complications, including death. Existing evidence suggests that the TLR/Myd88 signaling, which is upregulated in those with obesity, may contribute to the excessive inflammatory response seen in severe infection with SARS CoV-2 and other similar viral infections. Additional studies are needed to more firmly establish the role of increased TLR/MyD88 signaling in cells and tissues other than adipose in patients with obesity. Such exploration should be helpful beyond COVID-19, since TLR/MyD88 signaling in people with obesity could have a role in the development of chronic diseases, including heart disease and some types of cancer. Of course. while the TLR/MyD88 pathway is promising, it is also important to consider other potential targets for treatment for severe COVID-19 disease, especially in vulnerable individuals, such as those with obesity.

\section{Compliance with ethical standards}

Conflict of interest The authors declare that they have no conflict of interest.

Publisher's note Springer Nature remains neutral with regard to jurisdictional claims in published maps and institutional affiliations.

\section{References}

1. Wiersinga WJ, Rhodes A, Cheng AC, Peacock SJ, Prescott HC. Pathophysiology, transmission, diagnosis, and treatment of coronavirus disease 2019 (COVID-19) A review. JAMA. 2020. https://doi.org/10.1001/jama.2020.12839.

2. Li H, Liu L, Zhjang D, Xu J, Dai H, Tang N, et al. SARS-CoV-2 and viral sepsis: observations and hypotheses. Lancet. 2020;395: $1717-1520$

3. Williamson EJ, Walker AJ, Bhaskaran K, Bacon S, Bates C, Morton CE, et al. OpenSAFELY: factors associated with COVID19 death in 17 million patients. Nature. 2020. https://doi.org/10. 1038/s41586-020-2521-4.

4. Channappanavar R, Perlman S. Pathogenic human coronavirus infection: causes and consequences of cytokine storm and immunopathology. Semin Immunopathol. 2017;39:529-39.
5. Coperchini F, Chiovato L, Croce L, Magri F, Rotondi M. The cytokine storm in COVID-19: an overview of the involvement of the chemokine/chemokine receptor system. Cytokine Growth Factor Rev. 2020;53:25-32.

6. Gheblawi M, Wang K, Viveiros A, Nguyen Q, Zhong J, Turner $\mathrm{AT}$, et al. Angiotensin-converting enzyme 2: SARS-CoV-2 receptor and regulator of the renin-angiotensin system. Circ Res. 2020;126:1456-72.

7. Akira S, Takeda K. Toll-like receptor signaling. Nat Rev Immunol. 2004;4:499-511.

8. Li G, Fan Y, Lai Y, Han T, Li Z, Zhou P, et al. Coronavirus infections and immune responses. J Med Virol. 2020;92:424-32.

9. Moreno-Eutimio MA, López-Macías C, Pasteli-Palacios R. Bioinformatic analysis and identification of single-stranded RNA sequences recognized by TLR7/8 in the SARS-CoV-2 and MERSCoV genomes. Microbes Infection. 2020;22:226-9.

10. Choudhury A, Mukherjee A. In silico studies on the comparative characterization of the interactions of SARS-CoV-2 spike glycoprotein with ACE-2 receptor homologs and human TLRs. J Med Virol. 2020;1-9. https://doi.org/10.1002/jmv.25987.

11. Sohn KM, Lee S-G, Kim HJ, Cheon S, Jeong H, Lee J, et al. COVID-19 patients upregulate toll-like receptor-4 mediated inflammatory signaling that mimics bacterial sepsis. J Korean Med Sci. 2020;35:e343 https://doi.org/10.3346/jkms.2020.35.e343

12. Ellulu MS, Patimah I, Khaza'ai H, Rahmat A, Abed Y. Obesity and inflammation: the linking mechanism and the complications. Arch Med Sci. 2017;13:851-63.

13. Cuevas AM, Lazo M, Zuñiga I, Carrasco F, Potter JJ, Alvarez V, et al. Expression of MYD88 in adipose tissue of obese people: is there some role, in the development of metabolic syndrome? Metab Syndr Relat Disord. 2017;15:80-5.

14. Ahmad R, Al-Mass A, Arizado V, Al-Hubail A, Al-Ghimlas F, Al-Arouj M, et al. Elevated expression of the toll-like receptors 2 and 4 in obese individuals: its significance for obesity-induced inflammation. J Inflamm. 2012;9:48.

15. Zhou S, Wang G, Zhang W. Effect of TLR4/Myd88 signaling pathway on sepsis-associated acute respiratory distress syndrome in rats, via regulation of macrophage activation and inflammatory response. Exp Ther Med. 2018;15:3376-84.

16. Lin X, Kong J, Wu Q, Yang Y, Ji P. Effect of TLR4/ MyD88 signaling pathway on expression of IL-1 $\beta$ and TNF- $\alpha$ in synovial fibroblasts from temporomandibular joint exposed to lipopolysaccharide. Mediators Inflamm. 2015:329405. https://doi. org/10.1155/2015/329405.

17. Angelopoulou A Alexandris N, Konstantinou E, Mesiarakis K, Zanidis $\mathrm{C}$ et al. Imiquimod- A toll like receptor 7 agonist-Is an ideal option for management of COVID 19. Environ Res. 2020;188. https://doi.org/10.1016/j.envres.2020.109858.

18. Broering R, Montag M, Jiang M, Lu M, Sowa J-P, Kleinehr K, et al. Corticosteroids shift the toll-like receptor response pattern of primary-isolated murine liver cells from an inflammatory to an anti-inflammatory state. Int Immunol. 2011;23:537-44.

19. Zhou J, Wang J, Gu M-Y, Zhang S-Q, Chen S-L, Zhang X-W, et al. Effect of dexamethasone on TLR4 and MyD88 expression in monocytes of patients with tuberculous meningitis. Eur J Inflamm. 2017;15:107-12.

20. Horby P, Lim WS, Emberson JR, Mafham M, Bell JL, Linsell L. RECOVERY Collaborative Group et al. Dexamethasone in hospitalized patients with COVID-19-preliminary report. N Engl J Med. 2020. https://doi.org/10.1056/NEJMoa2021436.

21. Chansrichavala P, Chantharaksri U, Sritara P, Chaiyaroj SC. Atorvastatin attenuates TLR4-mediated NF-kappaB activation in a MyD88-dependent pathway. Asian Pac J Allergy Immunol. 2009;27:49-57. 